\title{
Long Sleep Duration is Associated with Increased High-Sensitivity C-Reactive Protein: A Nationwide Study on Chinese Population
}

This article was published in the following Dove Press journal: Diabetes, Metabolic Syndrome and Obesity: Targets and Therapy

\section{Liyun $\mathrm{He}$ \\ $\mathrm{Na}$ Yang \\ Fan Ping \\ Lingling $\mathrm{Xu}$ \\ Wei Li (ID \\ Yuxiu Li \\ Huabing Zhang (D)}

Department of Endocrinology, Key Laboratory of Endocrinology, Ministry of Health, Peking Union Medical College Hospital, Chinese Academy of Medical Sciences \& Peking Union Medical College, Beijing, People's Republic of China
Correspondence: Huabing Zhang Address: Peking Union Medical College Hospital, Chinese Academy of Medical Sciences \& Peking Union Medical College, Beijing, People's Republic of China Tel/Fax +86-0 I0-69I55073

Email huabingzhangchn@I63.com
Purpose: High-sensitivity C-reactive protein (hs-CRP) has been shown as an important predictor of cardiovascular risk, and sleep duration is an important factor influencing health outcomes. However, the association between hs-CRP and sleep duration among Chinese adults remained unknown and controversial. We aimed to explore the associations between sleep duration and hs-CRP levels among Chinese adults, and the differences in sex and geographical regions.

Methods: This cross-sectional study was based on nationally representative Chinese samples from the 2009 China Health and Nutrition Survey, which employed multistage, random cluster designs. Habitual sleep duration was self-reported with short and long sleep duration defined as $\leq 6 \mathrm{~h}$ and $\geq 9 \mathrm{~h}$ per day, respectively. Participants were divided into 4 groups based on the categories of self-reported sleep duration ( $\leq 6 \mathrm{~h}, 7 \mathrm{~h}, 8 \mathrm{~h}, \geq 9 \mathrm{~h}$ per day), excluding hsCRP levels $\geq 10 \mathrm{mg} / \mathrm{L}$. Subset samples were stratified by sex and geographical regions.

Results: A total of 8170 Chinese adults with a mean age of $50.0 \pm 14.9$ years and $4369(53.5 \%)$ men were included. Participants sleeping eight hours had the lowest hs-CRP levels. Long sleep duration $(\geq 9 \mathrm{~h})$ was significantly associated with elevated hs-CRP levels after adjusting for multiple covariates in full samples ( $\beta=0.0356$; $95 \%$ CI: $0.0050-0.0663$; $\mathrm{P}=0.0228)$, but the associations of short sleep duration $(\leq 6 \mathrm{~h})$ were limited to the unadjusted model. Long sleep duration was associated with elevated hs-CRP levels in women $(\beta=0.0512 ; 95 \%$ CI: $0.0097-0.0927$; $\mathrm{P}=0.0155)$ and in the northern region of China $(\beta=0.0699 ; 95 \%$ CI: $0.0210-0.1187 ; \mathrm{P}=0.0051)$, but not in men or the southern region.

Conclusion: Long but not short sleep duration was positively associated with elevated hsCRP levels, independent from traditional risk factors among Chinese adults, and sex and geographical differences were observed. Our findings imply that modifications of long sleep duration might be added to behavioral therapy to reduce cardiovascular risk in Chinese adults.

Keywords: high-sensitivity C-reactive protein, sleep duration, cardiovascular risk, Chinese population

\section{Introduction}

Studies on sleep are increasingly attracting universal attention. ${ }^{1,2}$ Previous studies have shown that sleep plays a critical role in health maintenance and is associated with numerous health outcomes such as aging, obesity, hypertension, stroke, and cardiovascular disease (CVD). ${ }^{3-6}$ Among the factors being closely associated with sleep problems, sleep duration is comparably the easiest to modify for the amelioration of health. C-reactive protein (CRP) is a typical biomarker for inflammation, 
and the high sensitivity CRP (hs-CRP) assay has low detection limits and good performance for critical health outcomes. ${ }^{7,8}$ Noticeably, hs-CRP is universally regarded as a more effective predictor of cardiovascular risk than traditional risk factors. ${ }^{9}$ Recently, emerging evidence has shown that anti-inflammatory therapy for patients with cardiovascular disease could significantly reduce the risk of cardiovascular events. ${ }^{10,11}$

Copious studies explored the associations between sleep duration and hs-CRP levels with inconsistent and controversial results. ${ }^{12-19}$ Linear $^{13-15}$ or curvilinear relationships $^{6}$ and $\mathrm{J}$-shaped ${ }^{12,13,16,18}$ or U-shaped associations $^{13,19}$ have been reported in studies. The differences in the measurements of sleep duration, the cutoff of increased hs-CRP, and targeted populations might account for the discrepancies to some extent. A previous meta-analysis, which included few studies on Chinese populations, was conducted with a high degree of heterogeneity. ${ }^{20}$ Most importantly, accumulating evidence indicates race/ethnicity differences in the relationships between sleep duration and hs-CRP levels. ${ }^{6,13}$ The existing large-scale studies were predominantly on Caucasian populations from Western countries; there have been no large-scale and nationally representative studies on Chinese populations. The conclusions from studies based on Caucasian-predominant populations might contain a great deal of incertitude for Chinese populations due to the differences in race/ethnicity. Some studies from Taiwan examined sleep duration and elevated hs-CRP levels, but their samples were too small and lacked generality. ${ }^{17,21}$ Thus, the exact relationships between sleep duration and hs-CRP in Chinese populations are still unclear. Since gender differences in the association of sleep duration and inflammations were reported in previous studies, ${ }^{13,18}$ whether the sexspecific effects modifications exist among Chinese adults remains unknown. Moreover, previous studies reported geographical region differences among adults living southern and northern region of China in the prevalence of hypertension $^{22}$ and obesity ${ }^{23}$ which were closely associated with inflammations. Considering the different lifestyle habits of people living in northern and southern regions of China, such as eating and sleep habits, there might be geographical region discrepancies in the association of sleep duration and inflammations.

Accordingly, the current cross-sectional study aimed to evaluate the association between self-reported sleep duration and hs-CRP levels nationwide among Chinese adults.
Many known confounders were taken into consideration. We also examined sex differences and geographical region discrepancies among Chinese adults.

\section{Methods}

\section{Study Population}

The China Health and Nutrition Survey (CHNS) was designed and conducted to examine the status of and changes in the health, nutrition, and family planning policies. ${ }^{24}$ The survey used a multistage, random cluster design to draw surveyed samples from nine provinces varying largely in geography, economic development, public resources, and health indicators. Valid informed consents were obtained from all the participants, and the surveys were approved by the Institutional Review Boards of the University of North Carolina at Chapel Hill and the National Institute for Nutrition and Health, Chinese Center for Disease Control and Prevention. More details and information about CHNS can be obtained elsewhere. ${ }^{25}$

Since blood samples were first collected in 2009, the present study focused only on the data from the 2009 CHNS. We excluded participants whose data were missing for values of sleep duration or hs-CRP concentrations, whose hs-CRP levels $\geq 10 \mathrm{mg} / \mathrm{L}$, and whose age was $<18$ years.

\section{Measurements}

\section{High-Sensitivity C-Reactive Protein}

Blood samples $(12 \mathrm{~mL})$ were collected from participants by venipuncture after an $8-12 \mathrm{~h}$ overnight fast. All blood samples were immediately centrifuged and stored at $-86^{\circ}$ $\mathrm{C}$ for later analyses, ultimately processed in a national central laboratory in Beijing (medical laboratory accreditation certificate ISO 15,189:2007) with strict quality control. The hs-CRP was quantified via the immunoturbidimetric method using an automated analyzer (Hitachi 7600, Tokyo, Japan) with Denka Seiken reagents (Tokyo, Japan).

Other biomarkers including triglycerides (TG), high density lipoprotein cholesterol (HDL-C), low density lipoprotein cholesterol (LDL-C), uric acid (UA), fasting serum glucose, and fasting serum insulin, previously described in detail for this sample, ${ }^{26}$ were also presented in our analysis.

\section{Sleep Duration}

Sleep duration was measured by a self-reported questionnaire. Participants were asked "how many hours do you usually spend in sleeping each day, including daytime and 
nighttime?". The responses about sleep duration were reported in only 1-hour increments and varied 1 to 18 $\mathrm{h}$ of sleep per day. Following recommendations from the American National Sleep Foundation as modified for Chinese adults, the optimal sleep duration was 7-8h per day, with short sleep duration defined as $\leq 6 \mathrm{~h}$ and long sleep duration defined as $\geq 9 \mathrm{~h}$ per day. ${ }^{27,28}$ Therefore, sleep duration was categorized into four groups: $\leq 6 \mathrm{~h}, 7 \mathrm{~h}, 8 \mathrm{~h}, \geq 9 \mathrm{~h}$.

\section{Sociodemographic Information and Anthropometry}

Age, sex, residence (rural or urban), educational attainment (low, medium, high), and household income per capita (categorized into tertiles as low, medium, and high) were investigated through the questionnaire. The nine provinces surveyed in 2009 were stratified into the northern region (Liaoning, Heilongjiang, Shandong, Henan) and southern region (Jiangsu, Hubei, Hunan, Guangxi, Guizhou), divided by the Qinling Mountain-Huaihe River Line. ${ }^{29}$ Anthropometry was conducted under standardized conditions, including measurements of height, weight, systolic blood pressure (SBP), and diastolic blood pressure (DBP). Body mass index (BMI) was calculated as weight $(\mathrm{kg})$ divided by the squared height in meters $\left(\mathrm{m}^{2}\right){ }^{30}$

\section{Lifestyle Factors and Health Status}

Lifestyle factors included physical activity (grouped into three levels: low, moderate, and high), total energy intake, smoking status (currently smoking or not), and alcohol drinking habits (currently drinking or not), ${ }^{26}$ which were collected through self-reported questionnaire. Health status of participants was also recorded in the CHNS interview by asking yes/no questions about participants' history of diagnosed diabetes and the use of oral antidiabetic drugs and insulin, their history of diagnostic hypertension and the use of antihypertensive drugs, and their history of diagnosed myocardial infarction (MI), stroke, or cancer.

\section{Statistical Analyses}

Characteristics were summarized with proportions for categorical variables and presented as mean \pm SD (standard deviance) for continuous variables. Differences between groups were determined by one-way analysis of variance or Kruskal-Wallis test for continuous variables and Pearson $\chi 2$ statistic for categorical variables. Hs-CRP levels were natural-log transformed prior to analysis to approach a normal distribution.
Relationships between CRP and categorical sleep duration were analyzed with generalized linear models (created by PROC GENMOD in SAS), using eight hours of sleep as the reference. Model 1 adjusted for age, sex, and BMI. Further adjustments in Model 2 included educational attainment, residence, geographical region, and household income per capita. Model 3 additionally included life habitual factors such as smoking status, alcohol drinking habits, physical activity level, and total energy intake. Model 4 included Model 3 covariates and SBP, DBP, TG, HDL-C, LDL-C, UA, fasting serum glucose, and fasting serum insulin. Model 5 contained Model 4 covariates and health status-related variables (mentioned above). Subgroup analyses were stratified by sex and geographical regions separately to explore differences and discrepancies. Sensitive analysis was conducted by categorizing hs-CRP levels into two groups, high or low levels, in complete samples. The cut-off point for the classification was $3 \mathrm{mg} / \mathrm{L}$ of hs-CRP. ${ }^{31}$ Logistic regressions were used to access the association between hs-CRP categories and sleep duration with odd ratio (OR) and 95\% confidential interval (CI). $\mathrm{P}<0 \quad 0.05$ (two-sided) was regarded as statistically significant, and all the statistical analyses were conducted with SAS 9.4 (SAS Institute, Inc., Cary, NC, USA).

\section{Results}

\section{Characteristics of Participants Selected}

There were 9549 participants who provided blood samples and anthropometric measures in this survey. After excluding those whose values of sleep duration $(n=167)$ or hsCRP concentrations $(n=52)$ were missing, whose hs-CRP levels $\geq 10 \mathrm{mg} / \mathrm{L}$ ( $\mathrm{n}=382$ ), and who were $<18$ years of age $(\mathrm{n}=778)$, a total of 8170 adults with a mean age of $50.0 \pm$ 14.9 years and $4369(53.5 \%)$ men were finally included for the current study. The characteristics of all participants across sleep duration categories are presented in Table 1. Among the 8170 Chinese adults, $8 \mathrm{~h}$ of sleep was the most frequent sleep duration, and participants sleeping 8 hours had the lowest hs-CRP levels. A substantial minority, $22.9 \%$ of participants, had long sleep duration $(\geq 9 \mathrm{~h})$ and $10.2 \%$ of participants had short sleep duration $(\leq 6 \mathrm{~h})$. Participants with short sleep duration $(\leq 6 \mathrm{~h})$ were more likely to be older, have higher education attainments and household income, higher levels of LDL-C, fasting blood glucose, SBP, and DBP, and higher levels of physical activity, but they were less likely to live in rural regions. 
Table I Characteristics of Participants Across Sleep Duration

\begin{tabular}{|c|c|c|c|c|c|c|}
\hline & $\begin{array}{l}\leq 6 h \\
(n=83 I)\end{array}$ & $\begin{array}{l}7 h \\
(n=1575)\end{array}$ & $\begin{array}{l}8 \mathrm{~h}(\text { Mean }) \\
(\mathrm{n}=3893)\end{array}$ & $\begin{array}{l}\geq 9 h \\
(n=\mid 87 I)\end{array}$ & P Value ${ }^{a}$ & $\begin{array}{l}\text { Total } \\
(n=8 \mid 70)\end{array}$ \\
\hline Age (years) & $57.1 \pm 13.9$ & $51.4 \pm 13.7$ & $47.8 \pm \mid 4.1$ & $50.4 \pm 17.0$ & $<0.0001$ & $50.0 \pm 14.9$ \\
\hline Male (n, \%) & $459(55.2 \%)$ & $842(53.5 \%)$ & $2082(53.5 \%)$ & $986(52.7 \%)$ & 0.6852 & $4369(53.5 \%)$ \\
\hline BMI $\left(\mathrm{kg} / \mathrm{m}^{2}\right)$ & $23.5 \pm 3.5$ & $23.6 \pm 3.5$ & $23.4 \pm 3.4$ & $23.1 \pm 3.4$ & 0.0002 & $23.4 \pm 3.4$ \\
\hline Rural region $(\mathrm{n}, \%)$ & $46 \mathrm{I}(55.5 \%)$ & $958(60.8 \%)$ & $2699(69.3 \%)$ & $1327(70.9 \%)$ & $<0.0001$ & $5445(66.6 \%)$ \\
\hline Educational attainment & & & & & $<0.0001$ & \\
\hline Low (n, \%) & $655(78.7 \%)$ & $1118(71.0 \%)$ & $287 \mid(73.7 \%)$ & $|55|(82.9 \%)$ & & $6195(75.8 \%)$ \\
\hline Medium (n, \%) & $143(\mid 7.2 \%)$ & $348(22.1 \%)$ & $795(20.4 \%)$ & $266(\mid 4.2 \%)$ & & $1522(19.0 \%)$ \\
\hline High (n, \%) & $33(4.1 \%)$ & $109(6.9 \%)$ & $227(5.9 \%)$ & $54(2.9 \%)$ & & $423(5.2 \%)$ \\
\hline Household income per capita & & & & & $<0.0001$ & \\
\hline Low (n, \%) & $55(6.71 \%)$ & $117(7.5 \%)$ & $359(9.3 \%)$ & $211(11.4 \%)$ & & $742(9.2 \%)$ \\
\hline Medium (n, \%) & $275(33.5 \%)$ & $49 \mid(31.5 \%)$ & $1354(35.1 \%)$ & $714(38.7 \%)$ & & $2834(25.1 \%)$ \\
\hline High (n, \%) & $490(59.8 \%)$ & $951(61.0 \%)$ & $2147(55.6 \%)$ & $919(49.8 \%)$ & & $4507(55.7 \%)$ \\
\hline Geographical region & & & & & $<0.0001$ & \\
\hline Northern region $(\mathrm{n}, \%)$ & $406(48.9 \%)$ & $702(44.6 \%)$ & $1692(43.5 \%)$ & $687(36.7 \%)$ & & $3847(42.7 \%)$ \\
\hline Southern region $(\mathrm{n}, \%)$ & $425(51.4 \%)$ & $873(55.4 \%)$ & $220 \mathrm{I}(56.5 \%)$ & $1184(63.3 \%)$ & & $4683(57.3 \%)$ \\
\hline Total energy intake $(\mathrm{kcal} / \mathrm{d})$ & $2030.6 \pm 717.3$ & $2134.8 \pm 652.1$ & $2162.7 \pm 641.5$ & $2122.8 \pm 686.8$ & $<0.0001$ & $2134.8 \pm 665$. \\
\hline Current smoker (n, \%) & $255(30.7 \%)$ & $42 \mathrm{I}(26.7 \%)$ & $1054(27.1 \%)$ & $506(27.0 \%)$ & 0.1583 & $2236(27.4 \%)$ \\
\hline Alcohol drinking $(\mathrm{n}, \%)$ & $285(34.3 \%)$ & $543(34.5 \%)$ & $1280(32.9 \%)$ & $577(30.8 \%)$ & 0.1066 & $2685(32.9 \%)$ \\
\hline Physical activity & & & & & $<0.0001$ & \\
\hline Low (n, \%) & $528(62.5 \%)$ & $90 \mathrm{I}(57.2 \%)$ & 1944(49.9\%) & $963(51.5 \%)$ & & $4336(53.1 \%)$ \\
\hline Moderate (\%) & $103(12.4 \%)$ & $244(15.5 \%)$ & $594(15.3 \%)$ & $238(12.7 \%)$ & & $1179(14.4 \%)$ \\
\hline High (n, \%) & $200(24.0 \%)$ & $430(27.3 \%)$ & $1455(34.8 \%)$ & $670(35.8 \%)$ & & $2655(36.5 \%)$ \\
\hline \multicolumn{7}{|l|}{ Medical history } \\
\hline Diabetes (n, \%) & $35(4.2 \%)$ & $46(2.9 \%)$ & $92(2.4 \%)$ & $60(3.2 \%)$ & 0.0205 & $233(2.9 \%)$ \\
\hline Hypertension (\%) & $170(20.5 \%)$ & $210(13.3 \%)$ & $405(10.4 \%)$ & $27 \mid(\mid 4.5 \%)$ & $<0.0001$ & $1056(12.9 \%)$ \\
\hline MI (n, \%) & $13(1.6 \%)$ & $20(1.3 \%)$ & $23(0.6 \%)$ & $22(1.2 \%)$ & 0.0100 & $78(1.0 \%)$ \\
\hline Stroke (n, \%) & $17(2.0 \%)$ & II (0.7\%) & $39(1.0 \%)$ & $39(2.1 \%)$ & 0.0002 & $106(1.3 \%)$ \\
\hline \multicolumn{7}{|l|}{ Blood pressure } \\
\hline $\mathrm{SBP}(\mathrm{mmHg})$ & $130.5 \pm 20.8$ & $125.7 \pm 18.9$ & $123.0 \pm 17.8$ & $124.0 \pm 20.0$ & $<0.0001$ & $124.7 \pm 19.0$ \\
\hline $\mathrm{DBP}(\mathrm{mmHg})$ & $82.7 \pm 11.5$ & $81.0 \pm 11.4$ & $80.2 \pm 11.0$ & $79.6 \pm 11.5$ & $<0.0001$ & $80.5 \pm 11.3$ \\
\hline Hs-CRP (mg/L) & $1.80 \pm 1.88$ & $1.70 \pm 1.88$ & $1.55 \pm 1.74$ & $1.73 \pm 1.90$ & $<0.0001$ & $1.64 \pm 1.82$ \\
\hline TG $(\mathrm{mmol} / \mathrm{L})$ & $1.78 \pm 1.46$ & $1.67 \pm 1.52$ & $1.68 \pm 1.52$ & $1.66 \pm 1.46$ & 0.0278 & $1.68 \pm 1.50$ \\
\hline LDL-C (mmol/L) & $3.11 \pm 0.97$ & $3.03 \pm 0.97$ & $2.95 \pm 0.99$ & $2.92 \pm 0.95$ & $<0.0001$ & $2.97 \pm 0.98$ \\
\hline HDL-C (mmol/L) & $1.45 \pm 0.39$ & $1.44 \pm 0.49$ & $1.44 \pm 0.55$ & $1.43 \pm 0.43$ & 0.2854 & $1.44 \pm 0.50$ \\
\hline UA $(\mu \mathrm{mol} / \mathrm{L})$ & $316.5 \pm 98.5$ & $306.8 \pm 110.9$ & $306.8 \pm 105.0$ & $307.9 \pm 110.1$ & 0.0408 & $308.1 \pm 106.7$ \\
\hline Glucose (mmol/L) & $5.5 I \pm 1.50$ & $5.37 \pm 1.34$ & $5.30 \pm 1.38$ & $5.33 \pm 1.47$ & $<0.0001$ & $5.34 \pm I .4 \mid$ \\
\hline Insulin (ulU/mL) & $|4.9| \pm 28.3 \mid$ & $13.78 \pm 19.04$ & $14.17 \pm 21.58$ & $14.89 \pm 26.44$ & 0.1055 & $14.33 \pm 23.10$ \\
\hline
\end{tabular}

Notes: Data are expressed as the mean $\pm \mathrm{SD}$ or as number (percentage). ${ }^{\mathrm{a}} \mathrm{P}$ value represents the significance of difference across sleep duration categories. $\mathrm{P}$ values were determined by one-way analysis of variance or Kruskal-Wallis test for continuous variables and Pearson $\chi 2$ statistic for categorical variables.

Abbreviations: BMI, body mass index; MI, Myocardial infarction; SBP, systolic blood pressure; DBP, diastolic blood pressure; hs-CRP, high sensitive C-reactive protein; TG, triglycerides; LDL-C, low density lipoprotein cholesterol; HDL-C, high density lipoprotein cholesterol; UA, uric acid. Glucose: Fasting serum glucose. Insulin: Fasting serum insulin.

The characteristics of participants by sex and geographical region are displayed in Table 2. Men tended to report currently smoking and drinking, high physical activity levels, history of diabetes and stroke, higher total energy intake, and higher levels of TG, UA, SBP, and DBP, whereas women were more likely to report low education and income 
Table 2 Characteristics of Participants by Sex and Geographical Region

\begin{tabular}{|c|c|c|c|c|c|c|}
\hline & $\begin{array}{l}\text { Male } \\
(n=380 I)\end{array}$ & $\begin{array}{l}\text { Female } \\
(n=4369)\end{array}$ & $\mathbf{P}_{\text {Value }} \mathbf{a}^{\mathbf{a}}$ & $\begin{array}{l}\text { Northern } \\
(n=3487)\end{array}$ & $\begin{array}{l}\text { Southern } \\
(n=4683)\end{array}$ & P Value ${ }^{a}$ \\
\hline Sleep duration (hours) & $8.0 \pm 1.2$ & $7.9 \pm 1.2$ & 0.3070 & $7.8 \pm 1.2$ & $8.0 \pm 1.2$ & $<0.0001$ \\
\hline Sleep duration $\leq 6 \mathrm{~h}$ & $372(9.8 \%)$ & $459(10.5 \%)$ & & $406(11.7 \%)$ & $425(9.1 \%)$ & \\
\hline Sleep duration $=7 \mathrm{~h}$ & $733(19.3 \%)$ & $842(19.3 \%)$ & & $702(20.1 \%)$ & $873(18.6 \%)$ & \\
\hline Sleep duration $=8 \mathrm{~h}$ & $|8| I(47.7 \%)$ & $2082(47.7 \%)$ & & $1692(48.5 \%)$ & $220 I(47.0 \%)$ & \\
\hline Sleep duration $\geq 9 \mathrm{~h}$ & $885(22.6 \%)$ & $986(23.3 \%)$ & & $687(19.7 \%)$ & $1184(25.3 \%)$ & \\
\hline Age (years) & $50.0 \pm 15.0$ & $50.1 \pm 15.0$ & 0.9800 & $49.7 \pm 15.5$ & $50.3 \pm 15.3$ & 0.0814 \\
\hline BMI $\left(\mathrm{kg} / \mathrm{m}^{2}\right)$ & $23.4 \pm 3.4$ & $23.4 \pm 3.5$ & 0.8410 & $24.1 \pm 3.4$ & $22.8 \pm 3.3$ & $<0.0001$ \\
\hline Rural region (n, \%) & $2537(66.7 \%)$ & $2908(66.6 \%)$ & 0.8692 & $2385(68.4 \%)$ & $3060(65.3 \%)$ & 0.0038 \\
\hline Household income per capita & & & 0.0041 & & & 0.0115 \\
\hline Low $(n, \%)$ & $312(8.3 \%)$ & $430(10.0 \%)$ & & $350(10.1 \%)$ & $392(8.5 \%)$ & \\
\hline Medium (n, \%) & $1293(34.3 \%)$ & $154 \mid(35.7 \%)$ & & $1168(33.7 \%)$ & $1666(36.1 \%)$ & \\
\hline High $(n, \%)$ & $2165(57.4 \%)$ & $2342(54.3 \%)$ & & $1945(56.2 \%)$ & $2562(55.4 \%)$ & \\
\hline Educational attainment & & & $<0.0001$ & & & 0.0220 \\
\hline Low (n, \%) & $27 \mid 2(7 \mid .4 \%)$ & $3483(79.7 \%)$ & & $2619(75.1 \%)$ & $3576(76.3 \%)$ & \\
\hline Medium (n, \%) & $830(21.8 \%)$ & $722(16.5 \%)$ & & $660(18.9 \%)$ & $892(19.0 \%)$ & \\
\hline High (n, \%) & $259(6.8 \%)$ & $167(3.8 \%)$ & & $208(6.0 \%)$ & $215(4.6 \%)$ & \\
\hline Total energy intake (kcal/d) & $2338.0 \pm 669.7$ & $1957.9 \pm 609.9$ & $<0.0001$ & $2075.1 \pm 623.4$ & $2179.2 \pm 692.6$ & $<0.0001$ \\
\hline Current smoker (n, \%) & $2079(54.7 \%)$ & $157(3.6 \%)$ & $<0.0001$ & $938(26.9 \%)$ & I298(27.7\%) & 0.4124 \\
\hline Alcohol drinking (n, \%) & $2294(60.3 \%)$ & $391(9.0 \%)$ & $<0.0001$ & $1145(32.8 \%)$ & $1540(32.9 \%)$ & 0.9631 \\
\hline Physical activity & & & $<0.0001$ & & & $<0.0001$ \\
\hline Low (n, \%) & $1767(46.5 \%)$ & $2569(58.8 \%)$ & & $|84|(52.8 \%)$ & $2495(53.3 \%)$ & \\
\hline Moderate (n, \%) & $648(17.0 \%)$ & $531(12.1 \%)$ & & $446(\mid 2.8 \%)$ & $733(15.6 \%)$ & \\
\hline High (n, \%) & $1386(36.5 \%)$ & $1269(29.1 \%)$ & & $1200(34.4 \%)$ & $1455(31.1 \%)$ & \\
\hline \multicolumn{7}{|l|}{ Medical history } \\
\hline Diabetes (n, \%) & $125(3.3 \%)$ & $108(2.5 \%)$ & 0.0270 & $123(3.5 \%)$ & $110(2.4 \%)$ & 0.0015 \\
\hline Hypertension (n, \%) & $466(12.3 \%)$ & $590(13.5 \%)$ & 0.0945 & $462(13.2 \%)$ & $594(12.7 \%)$ & 0.4515 \\
\hline$M I(n, \%)$ & $38(1.0 \%)$ & $40(0.9 \%)$ & 0.6963 & $46(0.5 \%)$ & $32(0.4 \%)$ & 0.0035 \\
\hline Stroke $(\mathrm{n}, \%)$ & $74(1.9 \%)$ & $32(0.7 \%)$ & $<0.0001$ & $56(1.6 \%)$ & $50(1.1 \%)$ & 0.0335 \\
\hline \multicolumn{7}{|l|}{ Blood pressure } \\
\hline $\mathrm{SBP}(\mathrm{mmHg})$ & $126.1 \pm 17.5$ & $\mid 23.4 \pm 20.1$ & $<0.0001$ & $126.3 \pm 18.0$ & $123.5 \pm 19.4$ & $<0.0001$ \\
\hline $\mathrm{DBP}(\mathrm{mmHg})$ & $82.1 \pm 11.0$ & $79.0 \pm 11.3$ & $<0.0001$ & $82.8 \pm 11.1$ & $78.7 \pm 11.1$ & $<0.0001$ \\
\hline Hs-CRP (mg/L) & $1.68 \pm 1.81$ & $1.62 \pm 1.84$ & 0.0007 & $1.65 \pm 1.80$ & $1.64 \pm 1.85$ & 0.1696 \\
\hline TG $(\mathrm{mmol} / \mathrm{L})$ & $1.83 \pm 1.74$ & $1.56 \pm 1.25$ & $<0.0001$ & $1.75 \pm 1.66$ & $1.64 \pm 1.37$ & 0.0191 \\
\hline LDL (mmol/L) & $2.92 \pm 0.96$ & $3.02 \pm 0.98$ & $<0.0001$ & $3.04 \pm 1.03$ & $2.92 \pm 0.93$ & $<0.0001$ \\
\hline HDL (mmol/L) & $1.39 \pm 0.53$ & $1.48 \pm 0.47$ & $<0.0001$ & $1.42 \pm 0.56$ & $1.45 \pm 0.45$ & $<0.0001$ \\
\hline UA ( $\mu \mathrm{mol} / \mathrm{L})$ & $356.8 \pm 113.0$ & $265.7 \pm 79.6$ & $<0.0001$ & $292.3 \pm 107.1$ & $319.8 \pm 105.0$ & $<0.0001$ \\
\hline Glucose (mmol/L) & $5.4 I \pm 1.54$ & $5.28 \pm 1.27$ & $0.007 \mid$ & $5.50 \pm 1.56$ & $5.22 \pm 1.26$ & $<0.0001$ \\
\hline Insulin (ulU/mL) & $14.7 \pm 23.7$ & $14.0 \pm 22.5$ & 0.2633 & $15.0 \pm 22.5$ & $13.8 \pm 24.5$ & $<0.0001$ \\
\hline
\end{tabular}

Notes: Data are expressed as the mean \pm SD or as number (percentage). ${ }^{a} \mathrm{P}$ value represents the significance of group differences. $\mathrm{P}$ values were determined by 2 -side $t$-test for continuous variables and Pearson $\chi 2$ statistic for categorical variables.

Abbreviations: BMI, body mass index; MI, myocardial infarction; SBP, systolic blood pressure; DBP, diastolic blood pressure; hs-CRP, high sensitive C-reactive protein; TG, triglycerides; LDL-C, low density lipoprotein cholesterol; HDL-C, high density lipoprotein cholesterol; UA, uric acid. Glucose: Fasting serum glucose. Insulin: Fasting serum insulin.

attainments and higher levels of LDL-C. Participants in the northern region of China were more likely to have higher BMI, higher levels of TG, LDL-C, SBP, and DBP, lower levels of HDL-C, and reported a history of diabetes, MI, and stroke. Additionally, characteristics of participants across sleep duration categories were also reported for stratified 
samples separately (in Table S1 for men, Table S2 for women, Table S3 for the southern region, and Table S4 for the northern region).

\section{Associations Between Sleep Duration and $\mathrm{Hs}-\mathrm{CRP}$}

Results from multiple lineal regression analyses with multiple covariates adjustments in complete samples are displayed in Table 3. In the unadjusted model, both short $(\leq 6 \mathrm{~h})$ and long $(\geq 9$ h) sleep duration were significantly associated with hsCRP levels $(\mathrm{P}<0.05)$, but the associations between short sleep duration and hs-CRP levels disappeared with adjustments for age, sex, and BMI $(\mathrm{P}=0.5258)$. In contrast, long sleep duration retained its significant relationship with high hs-CRP levels in the full model ( $\beta=0.0369$ [95\% CI, 0.0062-0.0676]; $\mathrm{P}=0.0183$ ) including age, sex, $\mathrm{BMI}$, residence, educational attainment, household income per capita, geographical region, smoking, drinking, physical activity level, total energy intake, SBP, DBP, TG, HDL-C, LDL-C, UA, fasting glucose, fasting serum insulin, and even in the over-controlled model that additionally contained health status-related variables ( $\beta=0.0356$ [95\% CI, 0.0050-0.0663]; $\mathrm{P}=0.0228$ ).

\section{Subgroup Analyses by Sex and Geographical Regions}

Subgroup analyses by sex are displayed in Table 4. In female participants, the statistical significance of the associations between long sleep duration and high hs-CRP levels were continuously observed in all models ( $\beta=0.0512$ [95\% CI, 0.0097-0.0927]; $\mathrm{P}=0.0155$ ), whereas the associations with short sleep duration were not observed in all performing models $(\mathrm{P}>0.05)$. In male participants, however, neither short nor long sleep duration was significantly associated with hs-CRP levels.

When samples were stratified by geographical region, there were differences between the subgroups (Table 5). In the northern region of China, long sleep duration was positively associated with hs-CRP levels in all cases ( $\beta=0.0699$ [95\% CI, 0.0210-0.1187]; $\mathrm{P}=0.0051)$, while short sleep duration only showed significant associations in the unadjusted model. In the southern region of China, short sleep duration was positively related to hs-CRP levels only in the unadjusted models $(\mathrm{P}=0.0010)$, while long sleep duration was not associated with hs-CRP levels.

\section{Sensitivity Analysis}

The association between sleep duration and hs-CRP did not change while hs-CRP was treated as a categorical variable. Compared with $8 \mathrm{~h}$ of sleep duration, long sleep duration was significantly associated with high levels of hs-CRP $(\geq 3 \mathrm{mg} / \mathrm{L})$ in unadjusted model (OR=1.356 [95\% CI, 1.155-1.592]; $\mathrm{P}=0.0002)$ and multi-adjusted models (model 5: $\mathrm{OR}=1.259$ [95\% CI, 1.063-1.490]; $\mathrm{P}=0.0052$ ). Whereas, the association between short $(\leq 6 \mathrm{~h})$ sleep duration and high levels of hs-CRP $(\geq 3 \mathrm{mg} / \mathrm{L})$ was only observed in unadjusted model (OR=1.320 [95\% CI, 1.064-1.638]; $\mathrm{P}=0.0117)$, but not in multi-adjusted models (model 5: $\mathrm{OR}=1.007$ [95\% CI, 0.802-1.264]; $\mathrm{P}=0.9528$ ).

\section{Discussion}

This was the first large-scale, nationally representative study to evaluate the associations between hs-CRP and

Table 3 Associations Between Sleep Duration and Hs-CRP Levels in the Complete Samples

\begin{tabular}{|c|c|c|c|c|c|c|c|}
\hline \multirow[t]{2}{*}{ Model } & \multicolumn{2}{|l|}{$\leq 6 \mathrm{~h}$} & \multicolumn{2}{|l|}{ 7h } & \multirow{2}{*}{$\begin{array}{l}8 \mathrm{~h} \\
\text { [Reference] }\end{array}$} & \multicolumn{2}{|l|}{$\geq 9 \mathrm{~h}$} \\
\hline & $\beta(95 \% \mathrm{Cl})$ & P value & $\beta(95 \% \mathrm{Cl})$ & $P$ values & & $\beta(95 \% \mathrm{Cl})$ & $P$ value \\
\hline Participants, No. & \multicolumn{2}{|l|}{831} & \multicolumn{2}{|l|}{1575} & 3893 & \multicolumn{2}{|l|}{$187 \mid$} \\
\hline Unadjusted & $0.0957(0.0506,0.1408)$ & $<0.0001$ & $0.045 I(0.0098,0.0803)$ & 0.0122 & [Reference] & $0.0524(0.0192,0.0856)$ & 0.0020 \\
\hline Model I ${ }^{\mathrm{a}}$ & $0.0139(-0.0291,0.0570)$ & 0.5258 & $0.0064(-0.0268,0.0397)$ & 0.7051 & [Reference] & $0.0444(0.0131,0.0757)$ & 0.0054 \\
\hline Model $2^{b}$ & $0.0153(-0.0281,0.0588)$ & 0.4896 & $0.0093(-0.0242,0.0428)$ & 0.5859 & [Reference] & $0.042 \mathrm{I}(0.0105,0.0737)$ & 0.0090 \\
\hline Model $3^{c}$ & $0.0094(-0.0341,0.0528)$ & 0.6722 & $0.0080(-0.0254,0.0414)$ & 0.6388 & [Reference] & $0.0396(0.0082,0.0709)$ & 0.0134 \\
\hline Model $4^{\mathrm{d}}$ & $-0.0044(-0.0466,0.0379)$ & 0.8396 & $0.008 \mathrm{I}(-0.0224,0.0406)$ & 0.6247 & [Reference] & $0.0369(0.0062,0.0676)$ & 0.0183 \\
\hline Model $5^{e}$ & $-0.0049(-0.0472,0.0373)$ & 0.8194 & $0.084(-0.024 I, 0.0409)$ & 0.6113 & [Reference] & $0.0356(0.0050,0.0663)$ & 0.0228 \\
\hline
\end{tabular}

Notes: Hs-CRP was normalized by natural-log transformation; the level of hs-CRP at eight hours of sleep was used as a reference. $\beta$ ( $95 \%$ Cl): Regression coefficients and

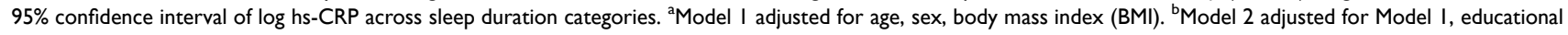
attainment, geographical region, residence and household income per capita. 'Model 3 adjusted for Model 2, smoking status, alcohol drinking habits, physical activities levels and total energy intake. 'Model 4 adjusted for Model 3, systolic and diastolic blood pressure, low density lipoprotein cholesterol (LDL-C), high density lipoprotein

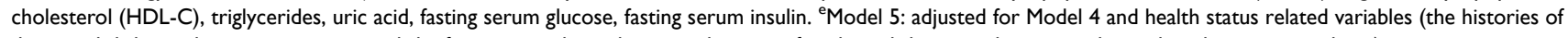
diagnosed diabetes, hypertension, myocardial infarction, stroke and cancer, the uses of oral antidiabetic medicines, insulin, and antihypertensive drugs).

Abbreviations: $\mathrm{Cl}$, confidence interval; hs-CRP, high-sensitivity C-reactive protein. 
Table 4 Relationships Between Sleep Duration and Hs-CRP Levels by Sex

\begin{tabular}{|c|c|c|c|c|c|c|c|}
\hline \multirow[t]{2}{*}{ Model } & \multicolumn{2}{|l|}{$\leq 6 \mathbf{h}$} & \multicolumn{2}{|l|}{ 7h } & \multirow{2}{*}{$\begin{array}{l}8 \mathrm{~h} \\
\text { [Reference] }\end{array}$} & \multicolumn{2}{|l|}{$\geq 9 \mathrm{~h}$} \\
\hline & $\beta(95 \% \mathrm{Cl})$ & $P$ value & $\beta(95 \% \mathrm{Cl})$ & $P$ value & & $\beta(95 \% \mathrm{Cl})$ & $P$ value \\
\hline \multicolumn{8}{|l|}{ Men } \\
\hline Participants, No. & \multicolumn{2}{|l|}{372} & \multicolumn{2}{|l|}{733} & 1811 & \multicolumn{2}{|l|}{885} \\
\hline Unadjusted & $0.0500(-0.0157,0.1157)$ & 0.1357 & $0.0386(-0.0119,0.0892)$ & 0.1338 & [Reference] & $0.0332(-0.0142,0.0805)$ & 0.1695 \\
\hline Model I ${ }^{\mathrm{a}}$ & $0.0024(-0.0616,0.0663)$ & 0.9424 & $0.0134(-0.0355,0.0622)$ & 0.5916 & [Reference] & $0.0297(-0.0160,0.0755)$ & 0.2028 \\
\hline Model $2^{b}$ & $0.0051(-0.0592,0.0695)$ & 0.8757 & $0.0148(-0.0342,0.0637)$ & 0.5548 & [Reference] & $0.0277(-0.0182,0.0736)$ & 0.2371 \\
\hline Model $3^{c}$ & $0.0017(-0.0625,0.0659)$ & 0.9592 & $0.0148(-0.0340,0.0637)$ & 0.5519 & [Reference] & $0.0258(-0.0200,0.0716)$ & 0.2690 \\
\hline Model $4^{d}$ & $-0.0006(-0.0626,0.0638)$ & 0.9851 & $0.0173(-0.0308,0.0653)$ & 0.4817 & [Reference] & $0.0227(-0.0224,0.0678)$ & 0.3238 \\
\hline Model $5^{\mathrm{e}}$ & $-0.0006(-0.0637,0.0625)$ & 0.9843 & $0.0200(-0.028 I, 0.068 I)$ & 0.4148 & [Reference] & $0.0214(-0.0238,0.0666)$ & 0.3530 \\
\hline \multicolumn{8}{|l|}{ Women } \\
\hline Participants, No. & \multicolumn{2}{|l|}{459} & \multicolumn{2}{|l|}{842} & 2082 & \multicolumn{2}{|l|}{986} \\
\hline Unadjusted & $0.1346(0.0726,0.1966)$ & $<0.001$ & $0.0507(0.0016,0.0997)$ & 0.0431 & [Reference] & $0.0688(0.0224,0.1153)$ & 0.0037 \\
\hline Model $\mathrm{I}^{\mathrm{a}}$ & $0.0180(-0.0401,0.0762)$ & 0.5433 & $-0.0008(-0.0461,0.0445)$ & 0.9731 & [Reference] & $0.0564(0.0136,0.0992)$ & 0.0097 \\
\hline Model $2^{b}$ & $0.0218(-0.0365,0.0801)$ & 0.4644 & $-0.0013(-0.0466,0.0440)$ & 0.9545 & [Reference] & $0.0549(0.0120,0.0978)$ & 0.0122 \\
\hline Model $3^{c}$ & $0.0159(-0.0426,0.0743)$ & 0.5941 & $-0.0040(-0.0492,0.0412)$ & 0.8619 & [Reference] & $0.0525(0.0096,0.0953)$ & 0.0163 \\
\hline Model $4^{d}$ & $-0.0102(-0.0667,0.0463)$ & 0.7232 & $0.0043(-0.0394,0.0479)$ & 0.8487 & [Reference] & $0.0517(0.0104,0.0931)$ & 0.0142 \\
\hline Model $5^{\mathrm{e}}$ & $-0.0087(-0.0654,0.0479)$ & 0.7626 & $0.0018(-0.0420,0.0456)$ & 0.9355 & [Reference] & $0.0512(0.0097,0.0927)$ & 0.0155 \\
\hline
\end{tabular}

Notes: Hs-CRP was normalized by natural-log transformation; the level of hs-CRP at eight hours of sleep was used as a reference. $\beta$ (95\% Cl): Regression coefficients and 95\% confidence interval of log hs-CRP across sleep duration categories. ${ }^{a}$ Model I adjusted for age, body mass index (BMI). ${ }^{b}$ Model 2 adjusted for Model I, educational attainment, geographical region, residence and household income per capita. 'Model 3 adjusted for Model 2, smoking status, alcohol drinking habits, physical activities levels and total energy intake. Model 4 adjusted for Model 3, systolic and diastolic blood pressure, low density lipoprotein cholesterol (LDL-C), high density lipoprotein cholesterol (HDL-C), triglycerides (TG), uric acid (UA), fasting serum glucose, fasting serum insulin. ${ }^{\mathrm{e}}$ Model 5 : adjusted for Model 4 and health status related variables (the histories of diagnosed diabetes, hypertension, myocardial infarction, stroke and cancer, the uses of oral antidiabetic medicines, insulin, and antihypertensive drugs).

Abbreviations: $\mathrm{Cl}$, confidence interval; hs-CRP, high-sensitivity $\mathrm{C}$-reactive protein.

estimated sleep duration among Chinese adults. Compared with eight hours of sleep, long sleep duration $(\geq 9 \mathrm{~h})$ was consistently associated with increased hs-CRP levels with adjustments of numerous confounders within complete samples. The effects of long sleep duration also existed in all models among women and participants from the northern region of China. Our findings highlighted the robustness of the associations and implied that long sleep duration might be an independent risk factor for elevated hs-CRP concentrations. Conversely, the associations of short sleep duration $(\leq 6 \mathrm{~h})$ with hs-CRP levels were limited to unadjusted models and disappeared with adjustments for age, sex, and BMI. Moreover, short sleepers were more likely to have inflammation-related risk factors in our study, such as older age, higher BMI, and higher levels of lipids, which suggested that the effects of short sleep duration in unadjusted models might largely be a result of these confounding factors.

\section{Race/Ethnicity Difference}

The American Academy of Sleep Medicine (AASM) and the Sleep Research Society (SRS) recommend 7-9h per night as the appropriate sleep duration for adults. ${ }^{32,33}$ For Chinese adults, 7-8h per day is recommended for optimal health and 9 hours or more are considered excessive sleep duration by the Chinese Center for Disease Control and Prevention, who summarized the features of Chinese populations and the recommendations from the American National Sleep Foundation. ${ }^{27,34}$ In our study, participants who slept eight hours regularly had the lowest levels of hsCRP, whereas long sleep was associated with high hs-CRP levels. A previous study from Taiwan claimed the opposite results, but the sample size was $353 .{ }^{17}$ However, another study from Taiwan ${ }^{21}$ showed results similar to our study. Moreover, several recent longitudinal studies found that long sleep duration independently elevated the incident risk to stroke $^{5}$ and mild cognition impairments ${ }^{35}$ in Chinese people and the risk of dementia in Japanese people, ${ }^{36}$ all related to inflammations. Furthermore, longer sleep duration was demonstrated to be associated with a higher risk of mortality among Chinese populations in a large-scale prospective study. ${ }^{37}$ Thus, the implications are that long sleep duration in Asian populations needs to be given much more attention. 
Table 5 Relationships Between Sleep Duration and Hs-CRP Levels by Geographical Region

\begin{tabular}{|c|c|c|c|c|c|c|c|}
\hline \multirow[t]{2}{*}{ Model } & \multicolumn{2}{|l|}{$\leq 6 \mathrm{~h}$} & \multicolumn{2}{|l|}{ 7h } & \multirow{2}{*}{$\begin{array}{l}8 \mathrm{~h} \\
\text { [Reference] }\end{array}$} & \multicolumn{2}{|l|}{$\geq 9 \mathrm{~h}$} \\
\hline & $\beta(95 \% \mathrm{Cl})$ & $P$ value & $\beta(95 \% \mathrm{Cl})$ & $P$ value & & $\beta(95 \% \mathrm{Cl})$ & $P$ value \\
\hline \multicolumn{8}{|c|}{ Southern Region } \\
\hline Participants, No. & \multicolumn{2}{|l|}{425} & \multicolumn{2}{|l|}{873} & 2201 & \multicolumn{2}{|l|}{1184} \\
\hline Unadjusted & $0.1067(0.0432,0.1703)$ & 0.0010 & $0.0421(-0.0059,0.0901)$ & 0.0853 & [Reference] & $0.0417(-0.0015,0.0849)$ & 0.0586 \\
\hline Model I ${ }^{\mathrm{a}}$ & $0.0018(-0.0583,0.0620)$ & 0.9523 & $-0.0032(-0.0480,0.0417)$ & 0.8902 & [Reference] & $0.0207(-0.0197,0.0611)$ & 0.3154 \\
\hline Model $2^{b}$ & $0.0038(-0.0566,0.064 I)$ & 0.9025 & $-0.0020(-0.0470,0.0429)$ & 0.9292 & [Reference] & $0.0197(-0.0208,0.0602)$ & 0.3407 \\
\hline Model $3^{c}$ & $-0.0009(-0.06 / 2,0.0593)$ & 0.9755 & $-0.0035(-0.0483,0.0413)$ & 0.8792 & [Reference] & $0.0210(-0.0194,0.0613)$ & 0.3090 \\
\hline Model $4^{d}$ & $-0.0105(-0.0694,0.0484)$ & 0.7279 & $-0.0032(-0.04692,0.0406)$ & 0.8872 & [Reference] & $0.0173(-0.0222,0.0568)$ & 0.3839 \\
\hline Model $5^{\mathrm{e}}$ & $-0.0107(-0.0696,0.0482)$ & 0.7695 & $-0.004 I(-0.0479,0.0398)$ & 0.8559 & [Reference] & $0.0166(-0.0229,0.0561)$ & 0.4098 \\
\hline \multicolumn{8}{|c|}{ Northern Region } \\
\hline Participants, No. & \multicolumn{2}{|l|}{406} & \multicolumn{2}{|l|}{702} & 1692 & \multicolumn{2}{|l|}{687} \\
\hline Unadjusted & $0.0827(0.0189,0.1465)$ & 0.0111 & $0.0484(-0.0034,0.1003)$ & 0.0671 & [Reference] & $0.0733(0.0211,0.1256)$ & 0.0029 \\
\hline Model $\mathrm{I}^{\mathrm{a}}$ & $0.0301(-0.0317,0.0918)$ & 0.3402 & $0.0181(-0.0314,0.0675)$ & 04738 & [Reference] & $0.0749(0.0253,0.1245)$ & 0.0031 \\
\hline Model $2^{b}$ & $0.0293(-0.0328,0.0914)$ & 0.3550 & $0.0164(-0.0333,0.0661)$ & 0.5173 & [Reference] & $0.0745(0.0246,0.1244)$ & 0.0034 \\
\hline Model $3^{c}$ & $0.0210(-0.0412,0.0831)$ & 0.5091 & $0.0156(-0.0340,0.0629)$ & 0.5705 & [Reference] & $0.0715(0.0216,0.1215)$ & 0.0050 \\
\hline Model $4^{\mathrm{d}}$ & $0.0036(-0.0572,0.0644)$ & 0.9086 & $0.0200(-0.0284,0.0684)$ & 0.4187 & [Reference] & $0.0717(0.0229,0.1205)$ & 0.0040 \\
\hline Model $5^{\mathrm{e}}$ & $0.0034(-0.0574,0.0642)$ & 0.9120 & $0.0210(-0.0274,0.0695)$ & 0.3953 & [Reference] & $0.0699(0.0210,0.1187)$ & 0.0051 \\
\hline
\end{tabular}

Notes: Hs-CRP was normalized by natural-log transformation; the level of hs-CRP at eight hours of sleep was used as a reference. $\beta$ ( $95 \%$ Cl): Regression coefficients and

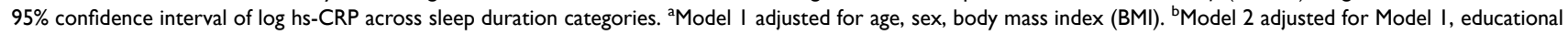
attainment, residence and household income per capita. 'Model 3 adjusted for Model 2, smoking status, alcohol drinking habits, physical activities levels and total energy intake. ${ }^{d}$ Model 4 adjusted for Model 3, systolic and diastolic blood pressure, low density lipoprotein cholesterol (LDL-C), high density lipoprotein cholesterol (HDL-C), triglycerides (TG), uric acid (UA), fasting serum glucose, fasting serum insulin. ${ }^{\mathrm{e}}$ Model 5: adjusted for Model 4 and health status related variables (the histories of diagnosed diabetes, hypertension, myocardial infarction, stroke and cancer, the uses of oral antidiabetic medicines, insulin, and antihypertensive drugs).

Abbreviations: $\mathrm{Cl}$, confidence interval; hs-CRP, high-sensitivity $\mathrm{C}$-reactive protein.

Noticeably, almost all previous studies based on predominantly Caucasian populations were different from the current study. Two previous studies reported null associations between hs-CRP levels and sleep duration after adjustment for covariates, but one with 43 non-pregnant young women in USA and the other with adults aged 30-60 years in UK. ${ }^{38,39}$ Alternatively, more studies elucidated apparent associations of short sleep duration with elevated CRP levels; ${ }^{12,13,16,18,19}$ some studies showed U-shaped relationships. ${ }^{13,19}$ The discrepancies mentioned above might largely result from the effects of race. Grandner and his colleague previously reported race differences but with predominantly White and few Asian participants. ${ }^{13}$ Our study with Chinese populations further supported the hypothesis of race difference in the effect of sleep duration on hs-CRP. Chinese populations are different from White populations in many aspects, ${ }^{40,41}$ including physiology and habitual lifestyles. Apart from different recommended optimal sleep duration, earlier studies demonstrated that the distributions of hs-CRP in Asian populations were different from those in Western populations. ${ }^{42} \mathrm{~A}$ recent scientific statement from AHA also elucidated the race discrepancies regarding the associations between sleep and cardiovascular risk factors. ${ }^{6}$

\section{Sex and Regional Difference}

Sex difference, possibly due to the effects of sex hormones ${ }^{43}$ varies in patterns with race. Our study found significant associations of long sleep duration with increased hs-CRP levels only among women, but null associations of sleep duration among men. On the contrary, a previous study on aging English people reported such associations among men, but not among women. ${ }^{44}$ Separately, the Whitehall study on British adults observed associations of short sleep duration only among women, but not among men. ${ }^{45}$ A previous study on American adults depicted linear relationships among women but J-shaped associations among men with a significant difference in long sleep duration. ${ }^{13}$ Another previous study on young American adults ${ }^{18}$ stated that elevated hs-CRP levels were associated with short sleep duration among men, while with long sleep duration among women. The differences in the findings of sex effects between our and other studies might be attributable to race effects, but the 
divergences among studies may have resulted from different targeted populations and measurements of sleep duration.

To the best of our knowledge, the regional differences between northern and southern China pertaining to sleep duration have never been reported before. Our study found continued associations of long sleep duration only in the northern region, while the relationships of short sleep duration were weak in the southern region. Studies reported a higher prevalence of obesity possibly owing to the difference in dietary patterns ${ }^{29,46}$ and a higher prevalence of hypertension probably associated with environmental exposure ${ }^{22}$ in the northern regions of China, such as different climates and latitudes. Consistent with those results, our study also showed higher BMI and more hypertension in participants from the northern regions of China. However, more studies are required to confirm the existence of geographical differences as well as to understand the underlying mechanisms.

\section{Implications}

CVD contains significant inflammatory components, ${ }^{9}$ and increasing evidence has established hs-CRP as an effective predictor for cardiovascular risk. ${ }^{7,9,47}$ As the burden of CVD in China is increasingly serious, ${ }^{48,49}$ there is an urgent need for effective CVD prevention strategies for Chinese populations. Appropriate management of sleep duration could contribute to the promotion of CVD prevention. Long sleep in association with increased hs-CRP levels probably needs to be given more attention with regard to cardiovascular risk. Recently, several studies have stated that long sleep duration was associated with an elevated risk of cardiovascular outcomes. ${ }^{33,50}$ Long sleep duration is possibly a behavioral risk factor for CVD, which has been largely ignored, especially in Asian populations.

Substantial studies elucidated a bidirectional connection between sleep and inflammation, in which the activation of the immune system might play an important role. ${ }^{1,2,51}$ Mechanisms explaining increased inflammation among short sleepers were explored in previous studies, ${ }^{4}$ while underlying mechanisms of long sleep duration remained poorly understood. Some studies assumed that long sleep duration was a predictor of participants with underlying conditions, especially inflammation-related diseases. ${ }^{1,33}$ However, more and further studies are required to better understand how long sleep duration influences health outcomes.

\section{Strengths and Limitations}

Our study is the first to evaluate the associations between sleep duration and hs-CRP levels in a largescale, nationwide sample of Chinese adults, which included more than 8000 participants from nine provinces in China. All known confounders were adjusted as much as possible in our study, including sociodemographic information, lifestyle factors, BMI, blood pressure, serum lipids levels, UA, fasting serum glucose, fasting serum insulin, and health status, ${ }^{6,8}$ which implied the possibility that long sleep duration might be an independent risk factor for high hs-CRP levels. Furthermore, hs-CRP represents a good prediction of cardiovascular risk beyond traditional risk factors, ${ }^{47}$ and sleep duration is a comparatively modifiable factor for health. Our study may inspire the modification of long sleep duration in targeted behavioral therapy to reduce CVD risks in Chinese adults.

Nevertheless, there were several limitations. First, as a cross-sectional study, the present study could not make causal inferences. Second, the polysomnography which is a valid tool for measuring sleep disorder is absent, and the self-bias is likely to have influenced self-reported sleep duration. Third, there may be bias due to the existence of unknown confounders. Fourth, participants who had concerns about sleep disturbance may be more likely to answer the questions about sleep duration and remember more clearly about their sleep duration.

\section{Conclusion}

This large-scale, nationally representative study of Chinese adults conclusively demonstrated a robust association between long sleep duration and high hs-CRP levels. This association was particularly strong among women, which indicated sex difference, and among participants in the northern region of China, which revealed the geographic regional difference. Short sleep duration was not associated with hs-CRP levels. Our findings were different from those in Western countries but appropriate for Chinese populations, which supports the possibility of race effects in the associations between sleep duration and hs-CRP levels. Our study suggested that long sleep duration might be a risk factor for CVD and simultaneously implied that modifications of long sleep duration might reduce the cardiovascular risk for Chinese adults. Further high-quality prospective and interventional studies are required to confirm our findings. 


\section{Abbreviations}

BMI, body mass index; CHNS, China Health and Nutrition Survey; CRP, C-reactive protein; CVD, cardiovascular disease; DBP, diastolic blood pressure; HDL-C, high density lipoprotein cholesterol; hs-CRP, high-sensitivity C-reactive protein; LDL-C, low density lipoprotein cholesterol; MI, myocardial infarction; SBP, systolic blood pressure; TG, triglycerides; UA, uric acid.

\section{Data Sharing Statement}

The datasets generated and/or analyzed during the current study are available in the CHNS repository, http://www. cpc.unc.edu/projects/china.

\section{Ethics Approval}

The China Health and Nutrition Survey (CHNS) is an ongoing open cohort, international collaborative project between the Carolina Population Center at the University of North Carolina at Chapel Hill and the National Institute for Nutrition and Health. ${ }^{24}$ The study was approved by the institutional review boards of the University of North Carolina at Chapel Hill and the National Institute for Nutrition and Health as well as the Chinese Center for Disease Control and Prevention.

\section{Acknowledgments}

All authors are grateful to the National Institute of Nutrition and Food Safety, China Center for Disease Control and Prevention, Carolina Population Center, The University of North Carolina at Chapel Hill and the Fogarty International Center for providing the data use. We also thank the China-Japan Friendship Hospital and the Ministry of Health for support for the CHNS 2009 survey. The authors are also grateful to the Staff of the Peking Union Medical College Hospital and all who actively participated in the study.

\section{Author Contributions}

Study concept and design: Zhang, He and Yang. Search of the relevant database and Quality assessment of each study: He, Yang and Zhang. Data extraction and confirmation of statistical analysis: He, Yang and Zhang. Statistical analysis: He, Zhang. Examination for the methodology: He and Zhang. Drafting of the manuscript: He and Zhang. Critical revision of the manuscript for important intellectual content: He, Yang, Xu, Ping, Wei Li, Zhang and Yuxiu Li. Obtained funding: Zhang. Administrative, technical, and material support: Zhang. Study supervision: Zhang. All authors have approved the final article. All authors made substantial contributions to conception and design, acquisition of data, or analysis and interpretation of data; took part in drafting the article or revising it critically for important intellectual content; agreed to submit to the current journal; gave final approval of the version to be published; and agree to be accountable for all aspects of the work.

\section{Funding}

The work was supported in part by the National Natural Science Foundation of China (91846106), Non-profit Central Research Institute Fund of Chinese Academy of Medical Sciences Clinical, and translational medicine research fund (2019XK320029). The Chinese Academy of Medical Sciences Innovation Fund for Medical Sciences (CIFMS2016-I2M-4-001), Training Program for Excellent Talents in Dongcheng District, and the Education Reforming Program, Peking Union Medical College (No. 2018zlgc0119) also funded the research. These organizations had no role in the design and conduct of the study; collection, management, analysis, and interpretation of the data; preparation, review, or approval of the manuscript; or the decision to submit the manuscript for publication.

\section{Disclosure}

The authors declare that they have no competing interests.

\section{References}

1. Irwin MR. Sleep and inflammation: partners in sickness and in health. Nat Rev Immunol. 2019;19(11):702-715. doi:10.1038/s41577-019-0190-z

2. Irwin MR. Why sleep is important for health: a psychoneuroimmunology perspective. Ann Rev Psychol. 2015;66:143-172. doi:10.1146/annurevpsych-010213-115205

3. Muscogiuri G, Barrea L, Annunziata G, et al. Obesity and sleep disturbance: the chicken or the egg? Crit Rev Food Sci Nutr. 2019;59(13):2158-2165. doi:10.1080/10408398.2018.1506979

4. Tobaldini E, Fiorelli EM, Solbiati M, Costantino G, Nobili L, Montano N. Short sleep duration and cardiometabolic risk: from pathophysiology to clinical evidence. Nat Rev Cardiol. 2019;16 (4):213-224. doi:10.1038/s41569-018-0109-6

5. Zhou L, Yu K, Yang L, et al. Sleep duration, midday napping, and sleep quality and incident stroke: the Dongfeng-Tongji cohort. Neurology. 2020;94(4):e345-e356. doi:10.1212/wnl.0000000000008739

6. St-Onge MP, Grandner MA, Brown D, et al. Sleep duration and quality: impact on lifestyle behaviors and cardiometabolic health: a scientific statement from the american heart association. Circulation. 2016;134 (18):e367-e386. doi:10.1161/cir.0000000000000444

7. Li Y, Zhong X, Cheng G, et al. Hs-CRP and all-cause, cardiovascular, and cancer mortality risk: A meta-analysis. Atherosclerosis. 2017;259:75-82. doi:10.1016/j.atherosclerosis.2017.02.003 
8. Tang Y, Liang $\mathrm{P}$, Chen J, et al. The baseline levels and risk factors for high-sensitive C-reactive protein in Chinese healthy population. Immunity Ageing. 2018;15:21. doi:10.1186/s12979-018-0126-7

9. Kaptoge S, Di Angelantonio E, Lowe G, et al. C-reactive protein concentration and risk of coronary heart disease, stroke, and mortality: an individual participant meta-analysis. Lancet. 2010;375 (9709):132-140. doi:10.1016/s0140-6736(09)61717-7

10. Tardif JC, Kouz S, Waters DD, et al. Efficacy and safety of low-dose colchicine after myocardial infarction. $N$ Engl J Med. 2019;381 (26):2497-2505. doi:10.1056/NEJMoa1912388

11. Ridker PM, Everett BM, Thuren T, et al. Antiinflammatory therapy with canakinumab for atherosclerotic disease. $N$ Engl $\mathrm{J}$ Med. 2017;377(12):1119-1131. doi:10.1056/NEJMoa1707914

12. Ferrie JE, Kivimaki M, Akbaraly TN, et al. Associations between change in sleep duration and inflammation: findings on C-reactive protein and interleukin 6 in the Whitehall II Study. Am J Epidemiol. 2013;178(6):956-961. doi:10.1093/aje/kwt072

13. Grandner MA, Buxton OM, Jackson N, Sands-Lincoln M, Pandey A, Jean-Louis G. Extreme sleep durations and increased C-reactive protein: effects of sex and ethnoracial group. Sleep. 2013;36 (5):769-779e. doi:10.5665/sleep.2646

14. Patel SR, Zhu X, Storfer-Isser A, et al. Sleep duration and biomarkers of inflammation. Sleep. 2009;32(2):200-204. doi:10.1093/sleep/ 32.2.200

15. Prather AA, Vogelzangs N, Penninx BW. Sleep duration, insomnia, and markers of systemic inflammation: results from the Netherlands Study of Depression and Anxiety (NESDA). J Psychiatr Res. 2015;60:95-102. doi:10.1016/j.jpsychires.2014.09.018

16. Holingue C, Owusu JT, Feder KA, Spira AP. Sleep duration and C-reactive protein: associations among pregnant and non-pregnant women. $J$ Reprod Immunol. 2018;128:9-15. doi:10.1016/j. jri.2018.05.003

17. Chiang JK. Short duration of sleep is associated with elevated high-sensitivity C-reactive protein level in Taiwanese adults: a cross-sectional study. J Clinical Sleep Medicine. 2014;10(7):743-749. doi: $10.5664 /$ jcsm.3862

18. Bakour C, Schwartz S, O’Rourke K, et al. Sleep duration trajectories and systemic inflammation in young adults: results from the national longitudinal study of adolescent to adult health (add health). Sleep. 2017;40(11):Nov. doi:10.1093/sleep/zsx156

19. Walsh CP, Lim A, Marsland AL, Ferrell RE, Manuck SB. Circulating Interleukin-6 concentration covaries inversely with self-reported sleep duration as a function of polymorphic variation in the glucocorticoid receptor. Brain Behav Immun. 2019;78:21-30. doi:10.1016/ j.bbi.2019.01.002

20. Irwin MR, Olmstead R, Carroll JE. Sleep disturbance, sleep duration, and inflammation: a systematic review and meta-analysis of cohort studies and experimental sleep deprivation. Biol Psychiatry. 2016;80 (1):40-52. doi:10.1016/j.biopsych.2015.05.014

21. Dowd JB, Goldman N, Weinstein M. Sleep duration, sleep quality, and biomarkers of inflammation in a Taiwanese population. Ann Epidemiol. 2011;21(11):799-806. doi:10.1016/j.annepidem.2011. 07.004

22. Pei L, Wu J, Wang Z, et al. Geographic variations and potential macro-environmental exposure of hypertension: from the China hypertension survey. J Hypertens. 2020;38(5):829-838. doi:10. 1097/hjh.0000000000002352

23. Zhang $\mathrm{X}$, Zhang M, Zhao Z, et al. Geographic variation in prevalence of adult obesity in China: results From the 2013-2014 national chronic disease and risk factor surveillance. Ann Intern Med. 2020;172(4):291-293. doi:10.7326/m19-0477

24. Han T, Zhang S, Duan W, et al. Eighteen-year alcohol consumption trajectories and their association with risk of type 2 diabetes and its related factors: the china health and nutrition survey. Diabetologia. 2019;62(6):970-980. doi:10.1007/s00125-019-4851-z
25. Popkin BM, Du S, Zhai F, Zhang B. Cohort profile: the China health and nutrition survey-monitoring and understanding socio-economic and health change in China, 1989-2011. Int J Epidemiol. 2010;39 (6):1435-1440. doi:10.1093/ije/dyp322

26. Liu X, Wu D, Qi X, et al. The associations between carbohydrate and protein intakes with habitual sleep duration among adults living in urban and rural areas. Clinical Nutrition. 2018;37(5):1631-1637. doi:10.1016/j.clnu.2017.07.024

27. Jin Donghui DS, Biyun C, Jiawu L, Zhongxi F, Huijun W. Changes on patterns of sleep duration: findingsfrom China health and nutrition survey in population in 9 provinces. Chinese J Epidemiology. 2016;37 (10):1366-1369. doi:10.3760/cma.j.issn.0254-6450.2016.10.010

28. Ning X, Lv J, Guo Y, et al. Association of sleep duration with weight gain and general and central obesity risk in Chinese adults: a prospective study. Obesity. 2020;28(2):468-474. doi:10.1002/oby.22713

29. Song F, Cho MS. Geography of food consumption patterns between South and North China. Foods. 2017;6(5):May. doi:10.3390/ foods 6050034

30. Dong F, Howard AG, Herring AH, et al. Longitudinal associations of away-from-home eating, snacking, screen time, and physical activity behaviors with cardiometabolic risk factors among Chinese children and their parents. Am J Clin Nutr. 2017;106(1):168-178. doi:10.3945/ ajcn.116.146217

31. Ridker PMA. Test in Context: high-Sensitivity C-Reactive Protein. J Am Coll Cardiol. 2016;67(6):712-723. doi:10.1016/j.jacc.2015.11.037

32. Watson NF, Badr MS, Belenky G, et al. Joint consensus statement of the american academy of sleep medicine and sleep research society on the recommended amount of sleep for a healthy adult: methodology and discussion. Sleep. 2015;38(8):1161-1183. doi:10.5665/sleep.4886

33. Wang C, Bangdiwala SI, Rangarajan S, et al. Association of estimated sleep duration and naps with mortality and cardiovascular events: a study of 116632 people from 21 countries. Eur Heart J. 2019;40(20):1620-1629. doi:10.1093/eurheartj/ehy695

34. CDC. Are you getting enough sleep?[EB/OL]. Available from: http:// www.cdc.gov/Features/Sleep/. Accessed February 15, 2016.

35. Zhu Q, Fan H, Zhang X, Ji C, Xia Y. Changes in sleep duration and 3 -year risk of mild cognitive impairment in Chinese older adults. Aging. 2020;12(1):309-317. doi:10.18632/aging.102616

36. Lu Y, Sugawara Y, Zhang S, Tomata Y, Tsuji I. Changes in sleep duration and the risk of incident dementia in the elderly Japanese: the Ohsaki cohort 2006 study. Sleep. 2018;41(10):Oct. doi:10.1093/sleep/zsy143

37. Cai H, Shu XO, Xiang YB, et al. Sleep duration and mortality: a prospective study of 113138 middle-aged and elderly Chinese men and women. Sleep. 2015;38(4):529-536. doi:10.5665/sleep.4564

38. Okun ML, Coussons-Read M, Hall M. Disturbed sleep is associated with increased C-reactive protein in young women. Brain Behav Immun. 2009;23(3):351-354. doi:10.1016/j.bbi.2008.10.008

39. Taheri S, Austin D, Lin L, Nieto FJ, Young T, Mignot E. Correlates of serum C-reactive protein (CRP)-no association with sleep duration or sleep disordered breathing. Sleep. 2007;30(8):991-996. doi:10.1093/sleep/30.8.991

40. Tsai CF, Thomas B, Sudlow CL. Epidemiology of stroke and its subtypes in Chinese vs white populations: a systematic review. Neurology. 2013;81(3):264-272. doi:10.1212/WNL.0b013e31829bfde3

41. Wu Z, Yao C, Zhao D, et al. Sino-MONICA project: a collaborative study on trends and determinants in cardiovascular diseases in China, Part i: morbidity and mortality monitoring. Circulation. 2001;103 (3):462-468. doi:10.1161/01.cir.103.3.462

42. Kelley-Hedgepeth A, Lloyd-Jones DM, Colvin A, et al. Ethnic differences in C-reactive protein concentrations. Clin Chem. 2008;54 (6):1027-1037. doi:10.1373/clinchem.2007.098996

43. Gaskins AJ, Wilchesky M, Mumford SL, et al. Endogenous reproductive hormones and C-reactive protein across the menstrual cycle: the BioCycle Study. Am J Epidemiol. 2012;175(5):423-431. doi:10.1093/aje/kwr343 
44. Jackowska M, Kumari M, Steptoe A. Sleep and biomarkers in the English Longitudinal Study of Ageing: associations with C-reactive protein, fibrinogen, dehydroepiandrosterone sulfate and hemoglobin. Psychoneuroendocrinology. 2013;38(9):1484-1493. doi:10.1016/j. psyneuen.2012.12.015

45. Miller MA, Kandala NB, Kivimaki M, et al. Gender differences in the cross-sectional relationships between sleep duration and markers of inflammation: whitehall II study. Sleep. 2009;32(7):857-864.

46. Zhang X, Zhang M, Zhao Z, et al. Geographic variation in prevalence of adult obesity in China: results from the 2013-2014 national chronic disease and risk factor surveillance. Ann Intern Med. 2019. doi:10.7326/m19-0477

47. Greenland P, Alpert JS, Beller GA, et al. 2010 ACCF/AHA guideline for assessment of cardiovascular risk in asymptomatic adults: a report of the American College of Cardiology Foundation/American Heart Association task force on practice guidelines. Circulation. 2010;122 (25):e584-636. doi:10.1161/CIR.0b013e3182051b4c
48. Yan S, Li J, Li S, et al. The expanding burden of cardiometabolic risk in China: the China health and nutrition survey. Obesity Rev. 2012;13 (9):810-821. doi:10.1111/j.1467-789X.2012.01016.x

49. Global, regional, and national age-sex specific mortality for 264 causes of death, 1980-2016: a systematic analysis for the global burden of disease study 2016. Lancet. 2017;390(10100):1151-1210. doi:10.1016/s0140-6736(17)32152-9

50. Jike M, Itani O, Watanabe N, Buysse DJ, Kaneita Y. Long sleep duration and health outcomes: A systematic review, meta-analysis and meta-regression. Sleep Med Rev. 2018;39:25-36. doi:10.1016/j. smrv.2017.06.011

51. Besedovsky L, Lange T, Haack M. The Sleep-Immune Crosstalk in Health and Disease. Physiol Rev. 2019;99(3):1325-1380. doi:10. 1152/physrev.00010.2018

\section{Publish your work in this journal}

Diabetes, Metabolic Syndrome and Obesity: Targets and Therapy is an international, peer-reviewed open-access journal committed to the rapid publication of the latest laboratory and clinical findings in the fields of diabetes, metabolic syndrome and obesity research. Original research, review, case reports, hypothesis formation, expert opinion and commentaries are all considered for publication. The manuscript management system is completely online and includes a very quick and fair peer-review system, which is all easy to use. Visit http://www.dovepress.com/testimonials.php to read real quotes from published authors. 